\title{
Galactic Cosmic Rays Anomalies
}

\section{Fiorenza Donato*}

Department of Physics, Torino University and INFN, Sezione di Torino

via Giuria 1, 10125 - Italy

E-mail: donatodto.infn.it

\begin{abstract}
Current measurements of galactic cosmic rays have reached an impressive precision for many species, and extend on wide energy ranges. Some species such as the high energy positron spectrum, the light primary and secondary nuclei and, possibly, the high energy part of the antiproton spectrum, are not straightforwardly explained by current models. The latter, indeed, are often calibrated on pre-existing cosmic data themselves. We outline how these anomalies, or effects in the data not straightforwardly explained by state-of-the-art theories, can open new doors in our understanding of the Galaxy and its particle components.
\end{abstract}

Neutrino Oscillation Workshop (NOW2018)

9 - 16 September, 2018

Rosa Marina (Ostuni, Brindisi, Italy)

${ }^{*}$ Speaker. 
A large contribution to the knowledge and understanding of the Galaxy is given by the observation of the most energetic particles, the cosmic rays (CRs). These relativistic particles, reaching the Earth from the outer space, are either primary nuclei, arriving directly from their sources, or secondary products of the spallation processes (i.e. fragmentation by nuclear destruction) taking place during the propagation from the sources through the interstellar medium (ISM). They include nuclei from hydrogen to very high $\mathrm{Z}$, and possibly in different isotopic compositions, electrons, antimatter (positrons and antiprotons measured up to now), and in some extensions also gamma rays. The main challenges we are facing are i) the identification of the sources of CRs, ii) the mechanism through which low energy particles, the seeds being single elements, dust or grains at rest in the ISM are accelerated to such high energy to be called CRs, and iii) the understanding of their propagation through the galactic magnetic fields.

Some rare components of the CRs, beside being secondaries, may also act as a peculiar means for the exploration of dark matter (DM) signals coming from the Galaxy. The most common hypothesis for particle DM consists of weakly interacting massive particles (WIMPs), supposed to be cold thermal relics after the Big Bang and to build up the galactic dark haloes. The socalled indirect DM investigation technique relies in the search for its stable annihilation products in the halo of galaxies, and in particular in the Milky Way. The indirect DM detection focuses on antiproton, antideuteron and positron in charged CRs, as well as in $\gamma$-rays and, more generally, on multiwavelength photons [1].

The most realistic propagation models are the diffusion ones, which account for spatial dependence of sources, CR densities and in principle of the ISM. The Galaxy is usually shaped as a thin gaseous disk where all the astrophysical sources are located, embedded in a thick diffusive magnetic halo. Diffusive models have proven to be successful in reproducing the nuclear, antiproton and radioactive isotopes data, in particular the ones collected by the Pamela and AMS-02 spaceborne detectors. These experiments have collected an unprecedented amount of data extending over decades of energy, and provided with errors often contained within few percent.

The most recent data have shown some anomalies, or effects not straightforwardly explained by state-of-the-art theories. Among them, a spectral break in the p, He, O, C primary nuclei has been measured by the Pamela and then confirmed by AMS-02 experiment $[2,3,4,5]$, with the $p$ spectrum following a slightly softer power law. Additionally, a break in the spectra of secondary nuclei has been detected by AMS-02 [6]. The rigidity dependence of Li, Be and B are nearly identical, but different from the primary $\mathrm{He}, \mathrm{C}$ and $\mathrm{O}$ (and also p). The discrepant harding between $\mathrm{p}$ and the other primary nuclei, and the other one between primary and secondary ones is not explained by standard propagation models, relying on isotropic diffusion and a single population of sources for all the CR hadrons.

The presence of a small amount of antimatter in CRs is predicted from spallation reactions of incoming protons and helium nuclei on the ISM (contributions from CRs with higher Z being negligible). The spallation products of these inelastic scatterings account for quarks and gluons, which immediately hadronize. In particular, the hadronization process includes the production of a small amount of antiprotons, antideuterons and positrons (the latter induced by decay processes). For antideuterons, only upper limits have been set but AMS-02. Indeed, the forthcoming GAPS experiment could be able to test some models for dark matter annihilation, predicting a sizable amount of low energy antideuterons [7]. 
The antiproton spectrum has been measured from below $\mathrm{GeV}$ up to about $400 \mathrm{GeV}$ energy $[8,9]$ with a sensitivity of few $\%$ over almost all the spectrum. The high energy tail of the data show a pretty hard behaviour, which can still be understood in terms of isotropic diffusion and secondary production of antiprotons, even if some caveats must be taken on the energy dependence of the diffusion coefficient. A source of large uncertainties resides in the production cross sections [10]. We emphasize the need of further high energy cross sections for a better understanding of CR data.

The positron fraction $e^{+} /\left(e^{+}+e^{-}\right)$has been measured by the PAMELA [11] satellite experiment and confirmed by the AMS-02 experiment [12], which also measures absolute leptons fluxes. It increases with energies, at variance with the predictions from pure secondary production of cosmic positrons. A viable explanation of the experimental result resides in the additional contribution of astrophysical sources accelerating leptons in their sites. The cosmic fluxes of positrons and leptons are quite sensitive to the presence of sources in the near Galaxy (few kpc), whose physics can be explored also in this peculiar channel [13] (and refs. therein).

Recently, the lepton data have also been analyzed in terms of their arrival direction $[14,13]$. Upper limits on the dipole anisotropy $\Delta$ have been set for the electron plus positron flux [15]. Indeed, the dipole anisotropy in the leptons arriving at the Earth can be a remarkable observable to constrain the properties of astrophysical emitters of $e^{+}$and $e^{-}$. As visible in Fig. 1, leptonic data can be interpreted with the emission of electrons and positrons from Supernova Remnants, Pulsar Wind Nebulae and the secondary production [13]. The anisotropy data are now close, or even lower than theoretical models.

In conclusion, recent data from space-based experiments has measured with unprecedented precision a wide number of CR species, from anti-matter to several nuclei in the Galaxy. In particular, the high energy positron spectrum, the light primary and secondary nuclei and, possibly, the high energy part of the antiproton spectrum, are not straightforwardly explained by state-of-the-art models. They require to go beyond the standard lore for the production and acceleration of galactic charged particles, invoking new hypothesis in the sources, in the diffusion in the Galaxy, in the distribution of the gas and in the cross sections regulating the production of secondary species.

\section{References}

[1] P. Salati, F. Donato, N. Fornengo Indirect dark matter detection with cosmic antimatter. Cambridge University Press 26 (2010) 521.

[2] O. Adriani et al., PAMELA Measurements of Cosmic-ray Proton and Helium Spectra, Science 332, (2011) 69 .

[3] M. Aguilar et al., Precision Measurement of the Proton Flux in Primary Cosmic Rays from Rigidity 1 GV to 1.8 TV with the Alpha Magnetic Spectrometer on the International Space Station Phys. Rev. Lett. 114, (2015) 171103.

[4] M. Aguilar et al., Precision Measurement of the Helium Flux in Primary Cosmic Rays of Rigidities 1.9 GV to 3 TV with the Alpha Magnetic Spectrometer on the International Space Station Phys. Rev. Lett. 115, (2015) 211101.

[5] M. Aguilar et al., Observation of the Identical Rigidity Dependence of He, C, and O Cosmic Rays at High Rigidities by the Alpha Magnetic Spectrometer on the International Space Station Phys. Rev. Lett. 119 (2017) 251101. 

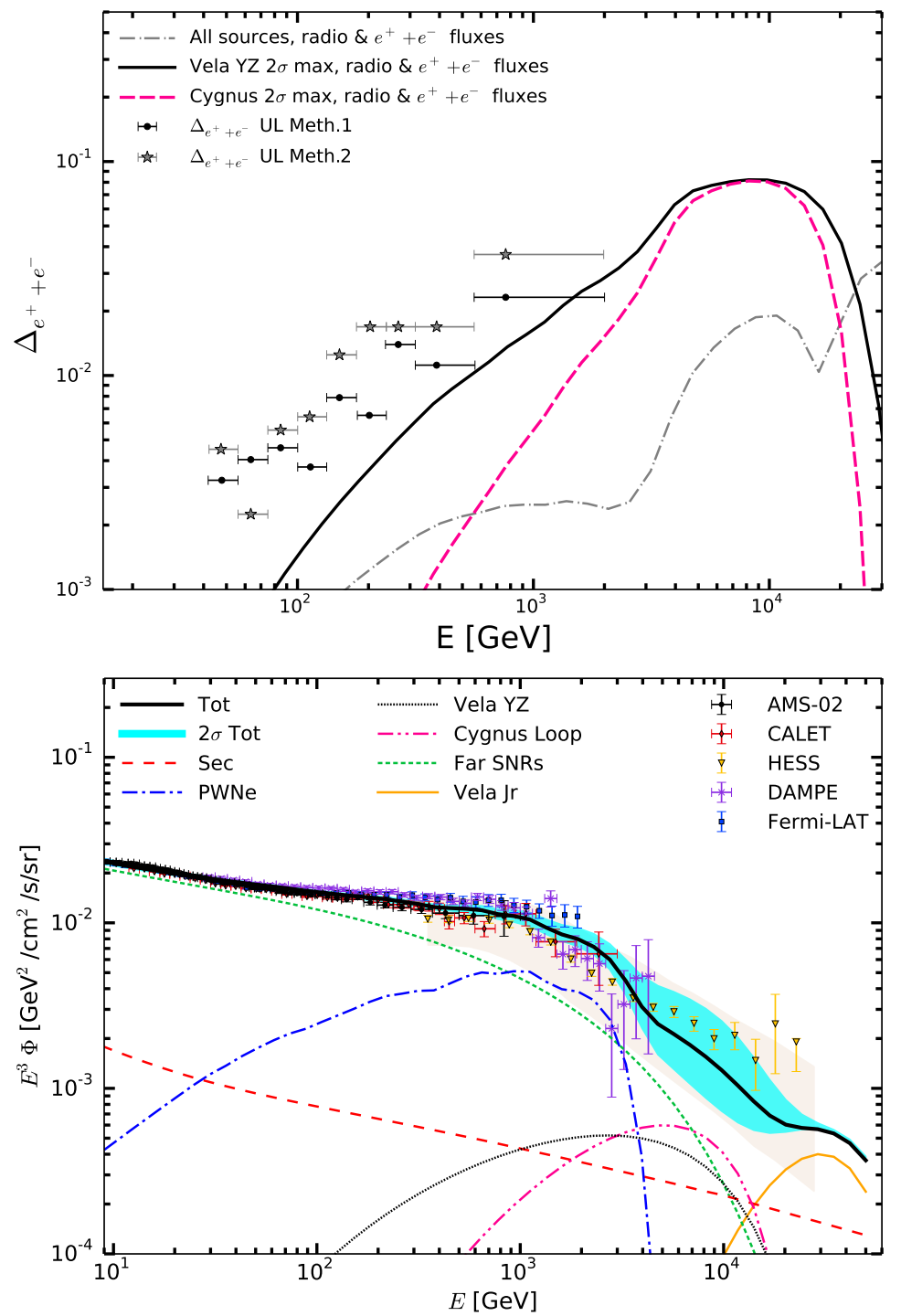

Figure 1: Results on the $e^{+}+e^{-}$flux (top) and on the corresponding dipole anisotropies (bottom) from the multi-wavelength fit to all the data. Left: The contribution from secondary production (red dashed), PWNe (blue dot dashed), Vela YZ (black dotted), Cygnus Loop (magenta dot-dot dashed), Vela Jr (orange solid) and the far smooth distribution of SNRs (green dotted) are shown. The $e^{+}+e^{-}$Fermi-LAT, AMS-02, DAMPE, HESS and CALET data with their statistics and systematic errors are also shown. Right: The maximal dipole anisotropy predicted for Vela YZ and Cygnus Loop as single dominant sources are reported with black solid and magenta dashed lines. The total anisotropy resulting from the distribution of all the sources is shown with gray dot-dashed line. Data on anisotropy from [15]. 
[6] M. Aguilar et al., Observation of New Properties of Secondary Cosmic Rays Lithium, Beryllium, and Boron by the Alpha Magnetic Spectrometer on the International Space Station Phys. Rev. Lett. 120 (2018) 021101.

[7] M. Korsmeier, F. Donato and N. Fornengo, Prospects to verify a possible dark matter hint in cosmic antiprotons with antideuterons and antihelium Phys. Rev. D 97, (2018) 103011.

[8] O. Adriani et al., PAMELA results on the cosmic-ray antiproton flux from $60 \mathrm{MeV}$ to $180 \mathrm{GeV}$ in kinetic energy Phys. Rev. Lett. 105, (2010) 121101.

[9] M. Aguilar et al., Antiproton Flux, Antiproton-to-Proton Flux Ratio, and Properties of Elementary Particle Fluxes in Primary Cosmic Rays Measured with the Alpha Magnetic Spectrometer on the International Space Station Phys. Rev. Lett. 117, (2016) 091103.

[10] M. Korsmeier, F. Donato and M. Di Mauro, Production cross sections of cosmic antiprotons in the light of new data from the NA61 and LHCb experiments Phys. Rev. D 97, (2018) 103019.

[11] O. Adriani et al., An anomalous positron abundance in cosmic rays with energies 1.5-100 GeV Nature 458, (2009) 607.

[12] M. Aguilar et al., Electron and Positron Fluxes in Primary Cosmic Rays Measured with the Alpha Magnetic Spectrometer on the International Space Station Phys. Rev. Lett. 113, (2014) 121102.

[13] S. Manconi, M. Di Mauro and F. Donato, Multi-messenger constraints to the local emission of cosmic-ray electrons arXiv:1803.01009 [astro-ph.HE], subm. to JCAP.

[14] S. Manconi, M. Di Mauro and F. Donato, Dipole anisotropy in cosmic electrons and positrons: inspection on local sources JCAP 1701, (2017) 006.

[15] S. Abdollahi et al., Search for Cosmic-Ray Electron and Positron Anisotropies with Seven Years of Fermi Large Area Telescope Data Phys. Rev. Lett. 118 (2017) 091103. 